Research Article

\title{
Traffic Status Dynamic Evaluation of an Eight-Lane Highway Based on the Lane Level
}

\author{
Jiayan Shen $\mathbb{D}^{1,2,3}$ Xiucheng Guo $\mathbb{D}^{1,2,3}$ Yiming Zhang $\mathbb{D}^{1,2,3}$ and Meina Zheng $\mathbb{D}^{1,2,3}$ \\ ${ }^{1}$ Jiangsu Key Laboratory of Urban ITS, Southeast University, Nanjing 211189, China \\ ${ }^{2}$ Jiangsu Province Collaborative Innovation Center of Modern Urban Traffic Technologies, Southeast University, \\ Nanjing 211189, China \\ ${ }^{3}$ School of Transportation, Southeast University, Nanjing 211189, China \\ Correspondence should be addressed to Xiucheng Guo; seuguo@163.com
}

Received 17 August 2020; Revised 21 October 2020; Accepted 17 November 2020; Published 3 December 2020

Academic Editor: Mahmoud Mesbah

Copyright (C) 2020 Jiayan Shen et al. This is an open access article distributed under the Creative Commons Attribution License, which permits unrestricted use, distribution, and reproduction in any medium, provided the original work is properly cited.

\begin{abstract}
Traffic status analysis is the basis for highway management and control. Most of the previous research studies were carried out from the perspective of road segments. The purpose of this paper is to analyze the difference in traffic operation characteristics of an eight-lane highway from the perspective of lanes. Lane saturation, average lane speed, and lane density were selected as traffic state evaluation indicators, and the concept of state space division was introduced to classify the traffic operation status of each lane. Then, a single-system multi-index dynamic comprehensive evaluation method was adopted to reflect the time-varying characteristics and the development trend of the operation status of each lane. The research results can provide the theoretical support for more refined highway management and improve the service level of the eight-lane highway.
\end{abstract}

\section{Introduction}

Highways play an important role in accelerating interregional passenger and cargo transportation and promoting regional, social, and economic development. The increasingly strengthened transportation links between regions have put forward higher requirements on highway capacity. Many important channels such as Shenyang-Dalian Highway, Shanghai-Nanjing Highway, and Beijing-Zhuhai Highway have been transformed from the original four-lane highway into an eight-lane highway. Compared with the four-lane highway, the eight-lane highway has a higher degree of freedom for vehicles, and the lane-changing behavior is more complicated. Moreover, due to the implementation of lane-dividing management and control strategies, the vehicles present different operating characteristics between lanes. To improve the operation and management level and alleviate the traffic congestion, it is necessary to conduct an in-depth analysis of the traffic status of the eight-lane highway to provide more refined management and control guidance to the highway managers.
A majority of research works have been dedicated to the traffic status analysis of the highway. The Highway Capacity Manual divided the traffic flow into six service levels to describe the traffic status of the highway, which is currently the most widely used evaluation standard in traffic engineering practice [1]. Wu established a new approach for modeling of fundamental diagrams. According to the model, the fundamental diagram can be represented as a superposition of four homogeneous traffic states [2]. Kerner proposed a three-phase traffic flow theory to describe the characteristics of different traffic flow states and the phase transition process between states and explained the temporal and spatial characteristics of congestion patterns on highways [3-6]. Xia and Chen developed a data-clustering methodology to define the flow phases using continuous traffic data obtained through detectors and divided the traffic into five states by selecting the traffic flow, loop occupancy, and speed as the clustering indicators $[7,8]$. Azimi and Zhang adopted $K$-means, fuzzy $C$-means, and CLARA (clustering large applications) to classify freeway traffic flow conditions based on flow characteristics and found that the 
traffic status classification result obtained by the $K$-means clustering method is the closest to the Highway Capacity Manual level-of-service criteria [9]. Xu et al. evaluated the impacts of traffic states on crash risks on freeways and found that the traffic status at the level of service (LOS) E had the highest crash potential followed by LOS F and D. The traffic status at LOS B and A had the lowest crash potential $[10,11]$. Khansari et al. investigated the car-following behavior in different lanes of a freeway in Iran. The results showed that closer lanes to the median have higher speed and lower headway, and the capacity difference between inner and outer lanes is from about $7 \%$ up to $24 \%$ [12]. An analysis of real data on a three-lane freeway was carried out by Duret et al. and showed that the fraction of the total flow in the median lane increases linearly, whereas the opposite trend is observed for the center lane and particularly for the shoulder lane [13]. Chu et al. modified the cell transmission model to depict the temporal-spatial evolution of traffic congestion on freeways and proposed two piecewise linear regression models to describe the relationship between flow and density [14]. Yang et al. adopted CNM (Clauset-Newman-Moore) Community Division Method of Complex Network to analyze traffic status information deeply implied from the regional road network traffic flow data to objectively determine the reasonable classification criteria for the regional traffic state [15]. Yang et al. presented a novel methodology for determining the overall highway safety level by integrating statistical analysis and analytic network process with set pair analysis, which accounted for both quantitative and qualitative factors that contribute to traffic safety [16]. Hui et al. applied phase space reconstruction theory and Lyapunov exponent to analyze the nonlinear character of traffic flow and constructed a new Volterra method based on model order reduction via quadratic-linear systems to predict short-time traffic flow of highways [17]. Lv et al. and Polson and Sokolov developed a deep learning approach to capture the nonlinear spatiotemporal effects due to transitions between free flow, breakdown, recovery, and congestion $[18,19]$. Cai et al., Sun et al., and Luo, et al. proposed a spatiotemporal traffic flow prediction method by improving the $k$-nearest neighbor model and obtained a better prediction performance and accuracy [20-22].

Many researchers have made efforts to evaluate the traffic status of highways, but they mainly researched from the perspective of the road section rather than the lane, which results in the traffic state differences between lanes not being well reflected, especially for eight-lane highways. The objective of this research is to develop a traffic status dynamic evaluation method to reveal different traffic state characteristics and predict the changing trend based on the lane level to provide more refined suggestions for the management and control of the eight-lane highway. In this paper, the traffic flow data of the Shanghai-Nanjing Highway are taken as the research object, and the rest of this paper is organized as follows. In Section 2, the lane traffic characteristics are analyzed. The evaluation indices are selected in Section 3. Section 4 proposes a dynamic comprehensive evaluation model to classify and rank the traffic status of the lanes. In Section 5, a case study of the Shanghai-Nanjing
Highway is carried out to verify the feasibility of the proposed method. Finally, the main conclusions are summarized in Section 6.

\section{Lane Traffic Characteristics' Analysis}

The video data and detector data of the Wuxi section of the Shanghai-Nanjing Highway are collected to statistically analyze the traffic characteristics of the four lanes on one side (the innermost lane is lane 1, and the outermost lane is lane 4). The Shanghai-Nanjing Highway adopts a lane-dividing management and control strategy. Lane 1 is set for small passenger cars, and the minimum and maximum speed limits are $110 \mathrm{~km} / \mathrm{h}$ and $120 \mathrm{~km} / \mathrm{h}$, respectively. Lane 2 is the passenger lane, and the minimum and maximum speed limits are $90 \mathrm{~km} / \mathrm{h}$ and $120 \mathrm{~km} / \mathrm{h}$, respectively. Lane 3 is a passenger and truck lane with a minimum speed limit of $80 \mathrm{~km} / \mathrm{h}$ and a maximum speed limit of $100 \mathrm{~km} / \mathrm{h}$. Lane 4 is a passenger and truck lane with a minimum speed limit of $60 \mathrm{~km} / \mathrm{h}$ and a maximum speed limit of $100 \mathrm{~km} / \mathrm{h}$. The fundamental three-dimensional diagrams of the flow-speeddensity of each lane are shown in Figure 1.

Taking lane 1 as an example, the fundamental threedimensional diagram can be, respectively, projected into two-dimensional graphs of speed-density, flow-density, and flow-speed, as shown in Figure 2. It can be seen from Figure 2 that when the density is close to 0 , the free speed is about $110 \mathrm{~km} / \mathrm{h}$, and when the speed is close to 0 , the jam density is about $101 \mathrm{pcu} / \mathrm{km}$. The maximum traffic flow is about $2659 \mathrm{pcu} / \mathrm{h}$, and the corresponding critical speed and optimum density are $89.4 \mathrm{~km} / \mathrm{h}$ and $30 \mathrm{pcu} / \mathrm{km}$, respectively. In the same way, the characteristic parameter values of lane 2, lane 3, and lane 4 can also be obtained, as shown in Table 1.

\section{Evaluation Index Selection}

The rationality of the index selection directly affects the evaluation results. Evaluation index selection should follow the principles of scientificity, operability, systematization, relative independence, and adaptability. Taking into account the differences in the traffic characteristics of each lane of the eight-lane highway, lane saturation, average lane speed, and lane density are selected as the evaluation indexes to reflect the traffic status of each lane. The definition and calculation formula of each index are as follows.

3.1. Lane Saturation. The lane saturation refers to the ratio of lane traffic flow to capacity, and its calculation formula is

$$
s=\frac{q}{C}
$$

where $s$ is the lane saturation, $q$ is the lane traffic flow, and $C$ is the lane capacity.

3.2. Average Lane Speed. When the observation length is constant, the average lane speed value is the harmonic mean value of the observed vehicle speed at the lane. The calculation formula is 


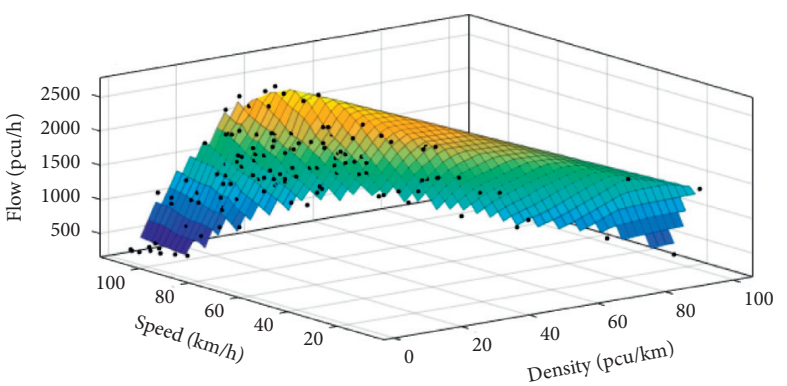

(a)

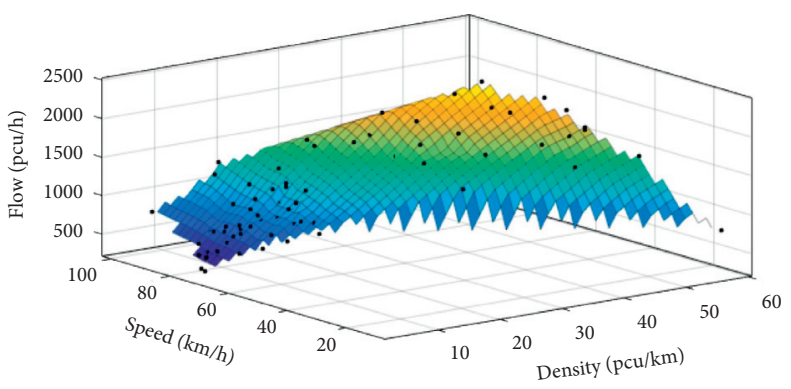

(c)

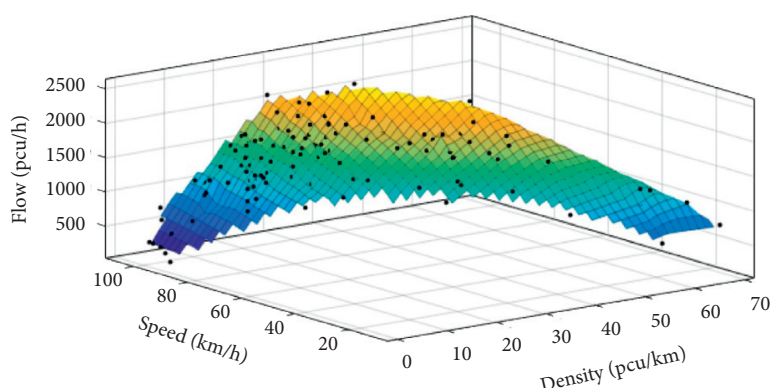

(b)

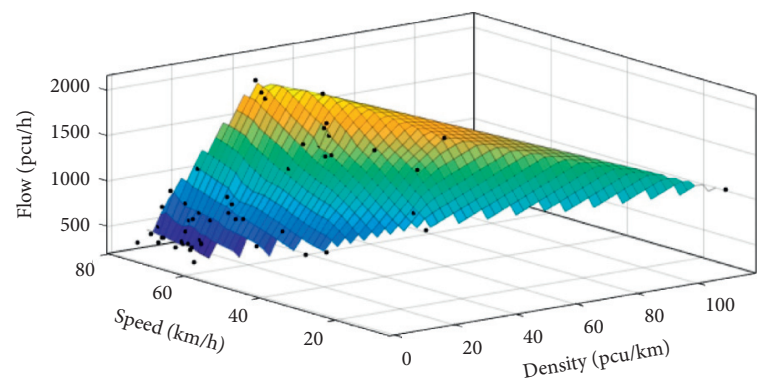

(d)

FIGURE 1: The flow-speed-density diagrams of each lane. (a) Lane 1. (b) Lane 2. (c) Lane 3. (d) Lane 4.

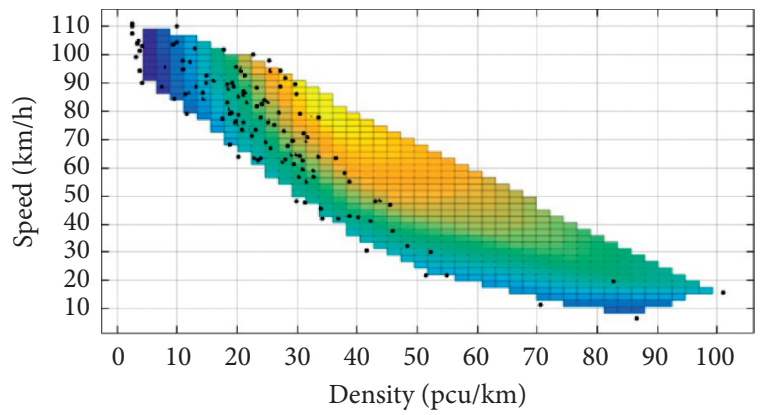

(a)

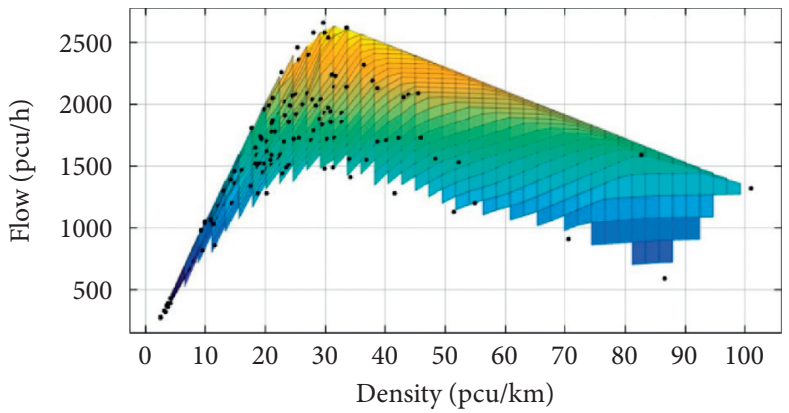

(b)

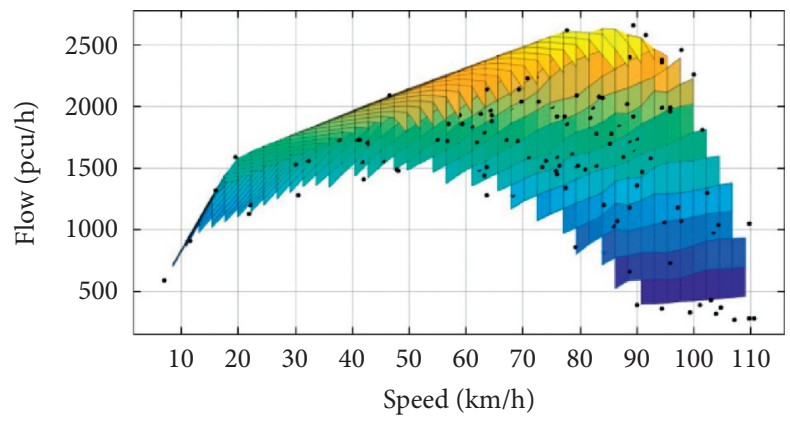

(c)

Figure 2: The two-dimensional graphs (speed-density, flow-density, and flow-speed) of lane 1. (a) Speed-density. (b) Flow-density. (c) Flow-speed.

$$
v=\frac{1}{(1 / p) \sum_{i=1}^{p}\left(1 / v_{i}\right)}=\frac{p x}{\sum_{i=1}^{p} t_{i}},
$$

where $v$ is the average lane speed, $x$ is the observation length, $p$ is the number of observation vehicles, $v_{i}$ is the location speed of the $i$ th vehicle, and $t_{i}$ is the travel time of the $i$ th vehicle. 
TABLE 1: Characteristic parameter values of each lane.

\begin{tabular}{lccccc}
\hline Lane & Maximum traffic flow $(\mathrm{pcu} / \mathrm{h})$ & Critical speed $(\mathrm{km} / \mathrm{h})$ & Optimal density $(\mathrm{pcu} / \mathrm{km})$ & Jam density $(\mathrm{pcu} / \mathrm{km})$ & Free speed $(\mathrm{km} / \mathrm{h})$ \\
\hline Lane 1 & 2659 & 89.4 & 30.0 & 101 & 110 \\
Lane 2 & 2520 & 79.2 & 31.5 & 69 & 102 \\
Lane 3 & 2420 & 58.4 & 41.6 & 58 & 100 \\
Lane 4 & 2072 & 67.7 & 31.4 & 114 & 77 \\
\hline
\end{tabular}

3.3. Lane Density. The lane density refers to the number of vehicles on one lane per unit length at a certain instant, and the calculation formula is

$$
k=\frac{D}{L}
$$

where $k$ is the lane density, $D$ is the number of vehicles in a section of a single lane, and $L$ is the section length.

\section{Methodology}

The traffic status of the highway changes dynamically in real time. General static evaluation models cannot fully reflect the real-time and dynamic properties. Therefore, a dynamic comprehensive evaluation method that adapts to different lane characteristics of the eight-lane highway is proposed with the evaluation idea of "first classification then ranking." That is, first classify and determine the traffic status and then sort and analyze the development process to carry out a multi-index dynamic comprehensive evaluation.

4.1. Index Preprocessing. When the index value is larger, the evaluation result is better. Such an index is called a positive index; otherwise, it is called an inverse index. Before the multi-index comprehensive evaluation calculation is performed, the indices need to be consistent. The conversion between positive indices and inverse indices is usually carried out in the form of reciprocal, as shown in the following equation:

$$
x_{i}^{\prime}= \begin{cases}\frac{1}{x_{i}} & \left(\text { if } x_{i}\right. \text { is an inverse index), } \\ x_{i} & \text { (if } x_{i} \text { is a positive index), }\end{cases}
$$

where $x_{i}^{\prime}$ is the converted index value and $x_{i}$ is the original index value.

Considering the inconsistency of the index dimensions, the extreme value method is used to nondimensionalize the indices to make the processed index value intervals fall into $[0,1]$. The formula is

$$
x_{i}^{*}=\frac{x_{i}^{\prime}-x_{i}^{\prime} \min }{x_{i}^{\prime} \max -x_{i}^{\prime} \min },
$$

where $x_{i}^{*}$ is the dimensionless index value, $x_{i}^{\prime}$ max is the maximum value of the $i$ th converted index, and $x_{i}^{\prime}$ min is the minimum value of the $i$ th converted index.

4.2. Classification Principle. The number of categories and classification standards should be determined appropriately according to the traffic operation of each lane of the eightlane highway. The concept of state space division is introduced in this paper to divide different traffic states. When the lane traffic status is divided into $N$ categories, the boundary threshold of each level is set as $\left\{X_{1}, X_{2}, \ldots, X_{N-1}\right\}$. If the preset boundary threshold index vectors $X_{i}=\left\{x_{i 1}, x_{i 2}, \ldots, x_{i n}\right\}^{T}(i=1,2, \ldots, N-1 ; n$ is the number of evaluation indexes) satisfy equation (6), the corresponding state space is $n$-dimensional. Taking $n=2$ as an example, the state space division is shown in Figure 3.

$$
\sum_{j=1}^{n}\left(x_{i j}\right)^{2}=\left(r_{i}\right)^{2}, \quad(i=1,2, \ldots, N-1)
$$

where $\left(r_{i}\right)^{2}$ is the comprehensive boundary threshold of each level.

At time $t$, set the preprocessed evaluation index vector as $x_{t}=\left(x_{t 1}, x_{t 2}, \ldots, x_{\mathrm{tn}}\right)^{T}$, and construct the evaluation function in the form of equation (7). Then, the traffic state classification can be determined by comparing the magnitude of $y$ and $\left(r_{i}\right)^{2}$. For example, when $y \leq\left(r_{1}\right)^{2}$, the lane traffic status belongs to level 1 . When $\left(r_{1}\right)^{2}<y \leq\left(r_{2}\right)^{2}$, the lane traffic status belongs to level 2. When $y>\left(r_{N-1}\right)^{2}$, the lane traffic status belongs to level $N$.

$$
y=\sum_{j=1}^{n}\left(x_{t j}\right)^{2} .
$$

4.3. Ranking Method. In order to more precisely reflect the dynamic development process of the traffic state, a singlesystem multi-index dynamic comprehensive evaluation method is adopted to rank the traffic operating state. Assuming that the index observation values $\left\{x_{j}\left(t_{k}\right)\right\}$ at time $t_{k}(k=1,2, \ldots, T)$ have been obtained, the evaluation function is constructed as follows:

$$
y\left(t_{k}\right)=\sum_{j=1}^{n} w_{j}\left(t_{k}\right) x_{j}\left(t_{k}\right), \quad(K=1,2, \ldots, T) .
$$

The value of the weight coefficient $w_{j}\left(t_{k}\right)$ directly affects the reliability and rationality of the evaluation results. To reduce the interference of subjective factors on the evaluation results, the "difference-driven" weighting method is adopted to determine the value of $w_{j}\left(t_{k}\right)$. The core idea of the "difference-driven" weighting method is that the weight coefficient reflects the degree of variation of the indicator in the entire indicator system and the degree of influence on other indicators. Therefore, the weight coefficient can be directly determined by the amount of information provided by each indicator. 


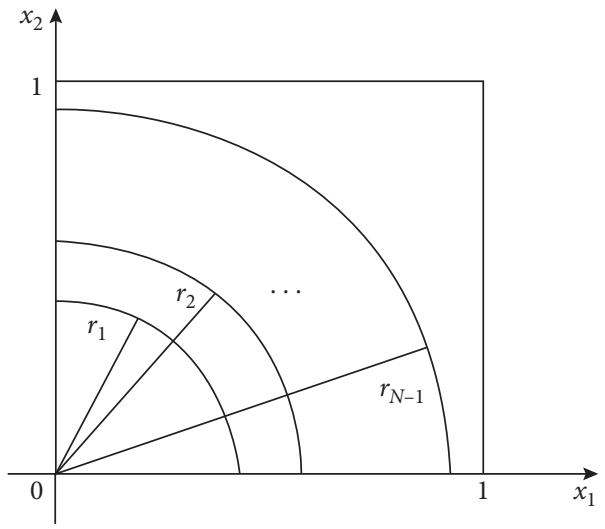

FIgURe 3: The state space division $(n=2)$

Set $A=\left[\begin{array}{cccc}x_{1}\left(t_{1}\right) & x_{2}\left(t_{1}\right) & \cdots & x_{n}\left(t_{1}\right) \\ x_{1}\left(t_{2}\right) & x_{2}\left(t_{2}\right) & \cdots & x_{n}\left(t_{2}\right) \\ \vdots & \vdots & \ddots & \vdots \\ x_{1}\left(t_{T}\right) & x_{2}\left(t_{T}\right) & \cdots & x_{n}\left(t_{T}\right)\end{array}\right]$ and $H=A^{T} A$.

Then, $Y=A w$ and $Y^{T} Y=w^{T} A^{T} A w=w^{T} H w$. When the original data are standardized, maximizing the sample variance of $Y$ is equivalent to maximizing $w^{T} H w$. The "difference-driven" weighting model can be constructed as shown in the following formula:

$$
\begin{array}{r}
\max \left\{w^{T} H w\right\}, \\
\text { s.t. } w^{T} w=1, \\
w>0,
\end{array}
$$

where

$$
H=A^{T} A=\left[\begin{array}{cccc}
\sum_{k=1}^{T} x_{1}^{2}\left(t_{k}\right) & \sum_{k=1}^{T} x_{1}\left(t_{k}\right) x_{2}\left(t_{k}\right) & \cdots & \sum_{k=1}^{T} x_{1}\left(t_{k}\right) x_{n}\left(t_{k}\right) \\
\sum_{k=1}^{T} x_{2}\left(t_{k}\right) x_{1}\left(t_{k}\right) & \sum_{k=1}^{T} x_{2}^{2}\left(t_{k}\right) & \cdots & \sum_{k=1}^{T} x_{2}\left(t_{k}\right) x_{n}\left(t_{k}\right) \\
\vdots & \vdots & \ddots & \vdots \\
\sum_{k=1}^{T} x_{n}\left(t_{k}\right) x_{1}\left(t_{k}\right) & \sum_{k=1}^{T} x_{n}\left(t_{k}\right) x_{2}\left(t_{k}\right) & \cdots & \sum_{k=1}^{T} x_{n}^{2}\left(t_{k}\right)
\end{array}\right] .
$$

When $H$ is a positive matrix, it has the only positive maximum eigenvalue and corresponding eigenvector. $w$ can be the standard eigenvector corresponding to the maximum eigenvalue of $H$ to obtain the maximum value of $w^{T} H w$.

4.4. Evaluation Process. The process of the dynamic comprehensive evaluation method mainly includes data acquisition, index preprocessing, traffic status classification, and ranking, as shown in Figure 4.

\section{Case Study}

5.1. Data Acquisition. The evaluation time segment of the traffic status can be determined according to the actual needs of the highway information system. The Wuxi section of the Shanghai-Nanjing Highway, as shown in Figure 5, was selected as the case study object. 15-minute index data including lane saturation, average lane speed, and lane density for each lane were collected from 9:00 am to 9:15 am on September 30, 2017. According to the vehicle type classification criterion, all vehicles on the Shanghai-Nanjing Highway were divided into four categories: small passenger cars, medium cars, large cars, and trailers. The vehicle conversion factors of these four categories are 1.0, 1.5, 2.5, and 4.0 , respectively. The entire 15 -minute index data were divided into 5 time periods with an evaluation interval of 3 minutes. The index data of each lane are shown in Table 2, where $s$ means the lane saturation, $v$ means the average lane speed, and $k$ means the lane density.

5.2. Index Preprocessing. Take the reciprocal of the average lane speed, and then perform dimensionless processing on all indexes. The evaluation index value processing results are shown in Table 3.

5.3. Traffic Status Classification. The traffic status of each lane is divided into six levels, and the fuzzy $C$-means method is used to determine the threshold values of the evaluation indicators of each level. After the nondimensional processing, the threshold values $\left\{X_{1}, X_{2}, X_{3}, X_{4}, X_{5}\right\}$ and the comprehensive boundary threshold values $\left(r_{i}\right)^{2}$ are shown in Table 4.

Taking the traffic operation state from the 12th to 15 th minutes as an example, when substituting the data into equation (7), the evaluation function values of each lane can be calculated as $y=[0.316,0.512,0.895,0.255]$. By comparing the magnitude of $y$ and $\left(r_{i}\right)^{2}$, it can be seen that lane 1 and lane 4 belong to the level 2 operation state. Lane 2 belongs to the level 3 operation state, and lane 3 belongs to the level 4 operation state. It means that, in the same time period, the lanes of the same cross section show different operating status levels. The operations of lane 2 and lane 3 are at a lower service level than lane 1 and lane 4 .

5.4. Traffic Status Ranking. Taking lane 1 as an example, the "difference-driven" weighting model can be solved by using MATLAB mathematical programming software, and the calculation results are as follows: 


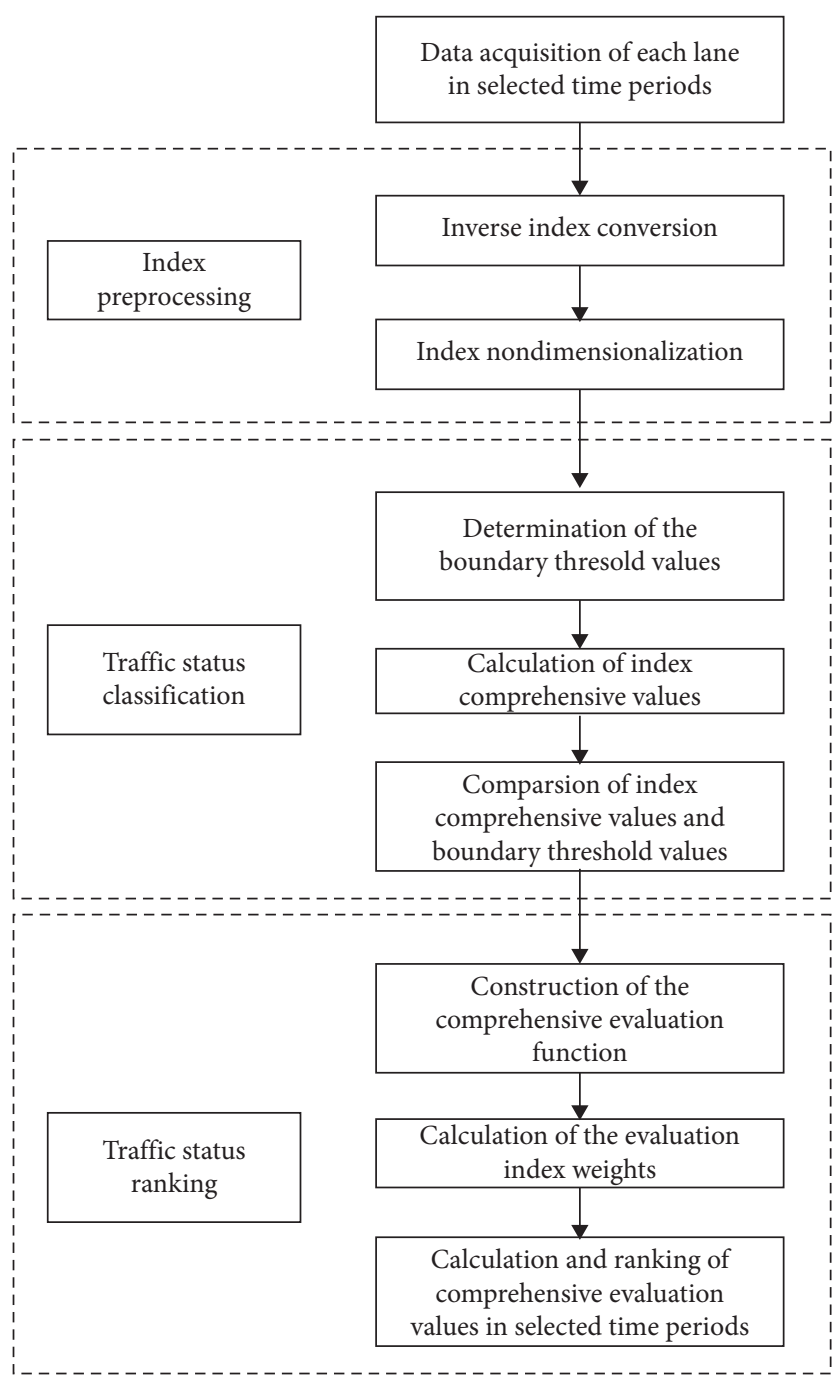

Figure 4: Process of the dynamic comprehensive evaluation method.

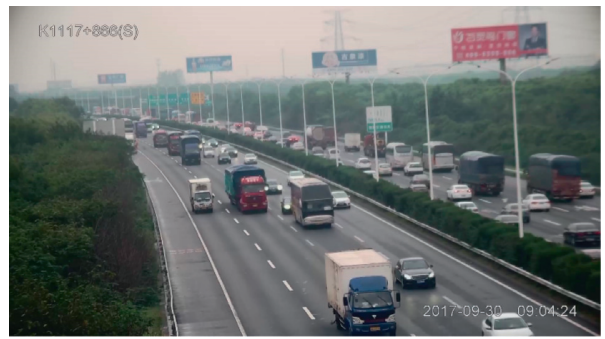

Figure 5: Wuxi section of the Shanghai-Nanjing Highway. 
TABLE 2: 15-minute index data for each lane.

\begin{tabular}{|c|c|c|c|c|c|c|}
\hline \multirow{2}{*}{ Time segment } & \multicolumn{3}{|c|}{ Lane 1} & \multicolumn{3}{|c|}{ Lane 2} \\
\hline & $s$ & $v(\mathrm{~km} / \mathrm{h})$ & $k(\mathrm{pcu} / \mathrm{km})$ & $s$ & $v(\mathrm{~km} / \mathrm{h})$ & $k(\mathrm{pcu} / \mathrm{km})$ \\
\hline $0 \sim 3 \mathrm{~min}$ & 0.41 & 81.93 & 13.43 & 0.49 & 86.64 & 14.13 \\
\hline $3 \sim 6 \mathrm{~min}$ & 0.43 & 82.02 & 13.90 & 0.48 & 92.55 & 13.01 \\
\hline $6 \sim 9 \min$ & 0.51 & 76.47 & 17.78 & 0.54 & 79.06 & 17.13 \\
\hline $9 \sim 12 \mathrm{~min}$ & 0.58 & 73.50 & 20.95 & 0.61 & 78.08 & 19.57 \\
\hline $12 \sim 15 \mathrm{~min}$ & 0.54 & 89.37 & 16.11 & 0.65 & 81.43 & 20.16 \\
\hline \multirow{2}{*}{ Time segment } & \multicolumn{3}{|c|}{ Lane 3} & \multicolumn{3}{|c|}{ Lane 4} \\
\hline & $s$ & $v(\mathrm{~km} / \mathrm{h})$ & $k(\mathrm{pcu} / \mathrm{km})$ & $s$ & $v(\mathrm{~km} / \mathrm{h})$ & $k(\mathrm{pcu} / \mathrm{km})$ \\
\hline $0 \sim 3 \mathrm{~min}$ & 0.76 & 73.18 & 25.12 & 0.54 & 70.70 & 15.84 \\
\hline $3 \sim 6 \mathrm{~min}$ & 0.58 & 82.83 & 17.05 & 0.56 & 60.45 & 19.19 \\
\hline $6 \sim 9 \mathrm{~min}$ & 0.75 & 67.55 & 26.74 & 0.69 & 43.32 & 33.24 \\
\hline $9 \sim 12 \mathrm{~min}$ & 0.73 & 65.07 & 27.23 & 0.71 & 55.81 & 26.52 \\
\hline $12 \sim 15 \mathrm{~min}$ & 0.82 & 73.78 & 26.84 & 0.48 & 63.68 & 15.70 \\
\hline
\end{tabular}

TABLE 3: Evaluation index value dimensionless processing results.

\begin{tabular}{|c|c|c|c|c|c|c|}
\hline \multirow{2}{*}{ Time segment } & \multicolumn{3}{|c|}{ Lane 1} & \multicolumn{3}{|c|}{ Lane 2} \\
\hline & $s$ & $v$ & $k$ & $s$ & $v$ & $k$ \\
\hline $0 \sim 3 \mathrm{~min}$ & 0.41 & 0.076 & 0.122 & 0.49 & 0.043 & 0.205 \\
\hline $3 \sim 6 \min$ & 0.43 & 0.076 & 0.126 & 0.48 & 0.025 & 0.189 \\
\hline $6 \sim 9 \mathrm{~min}$ & 0.51 & 0.097 & 0.162 & 0.54 & 0.071 & 0.248 \\
\hline $9 \sim 12 \mathrm{~min}$ & 0.58 & 0.110 & 0.190 & 0.61 & 0.075 & 0.284 \\
\hline $12 \sim 15 \mathrm{~min}$ & 0.54 & 0.051 & 0.146 & 0.65 & 0.062 & 0.292 \\
\hline \multirow{2}{*}{ Time segment } & \multicolumn{3}{|c|}{ Lane 3} & \multicolumn{3}{|c|}{ Lane 4} \\
\hline & $s$ & $v$ & $k$ & $s$ & $v$ & $k$ \\
\hline $0 \sim 3 \mathrm{~min}$ & 0.76 & 0.092 & 0.433 & 0.54 & 0.031 & 0.139 \\
\hline $3 \sim 6 \mathrm{~min}$ & 0.58 & 0.052 & 0.294 & 0.56 & 0.096 & 0.168 \\
\hline $6 \sim 9 \mathrm{~min}$ & 0.75 & 0.120 & 0.461 & 0.69 & 0.273 & 0.292 \\
\hline $9 \sim 12 \min$ & 0.73 & 0.134 & 0.469 & 0.71 & 0.133 & 0.233 \\
\hline $12 \sim 15 \mathrm{~min}$ & 0.82 & 0.089 & 0.463 & 0.48 & 0.073 & 0.138 \\
\hline
\end{tabular}

TABLE 4: Dimensionless and comprehensive boundary threshold values of evaluation indexes of each level.

\begin{tabular}{lccccc}
\hline Evaluation index & $X_{1}$ & $X_{2}$ & $X_{3}$ & $X_{4}$ & $X_{5}$ \\
\hline$s$ & 0.35 & 0.55 & 0.75 & 0.9 & 1 \\
$v$ & 0.035 & 0.083 & 0.154 & 0.267 & 0.593 \\
$k$ & 0.073 & 0.164 & 0.255 & 0.409 & 0.727 \\
$\left(r_{i}\right)^{2}$ & 0.129 & 0.336 & 0.651 & 1.049 & 1.880 \\
\hline
\end{tabular}

$A_{1}=\left[\begin{array}{cccc}x_{1}\left(t_{1}\right) & x_{2}\left(t_{1}\right) & \cdots & x_{n}\left(t_{1}\right) \\ x_{1}\left(t_{2}\right) & x_{2}\left(t_{2}\right) & \cdots & x_{n}\left(t_{2}\right) \\ \vdots & \vdots & \ddots & \vdots \\ x_{1}\left(t_{T}\right) & x_{2}\left(t_{T}\right) & \cdots & x_{n}\left(t_{T}\right)\end{array}\right]=\left[\begin{array}{ccc}0.410 & 0.076 & 0.122 \\ 0.430 & 0.076 & 0.126 \\ 0.510 & 0.097 & 0.162 \\ 0.580 & 0.110 & 0.190 \\ 0.540 & 0.051 & 0.146\end{array}\right]$

$H_{1}=A_{1}^{T} A_{1}=\left[\begin{array}{lll}1.241 & 0.205 & 0.376 \\ 0.205 & 0.036 & 0.063 \\ 0.376 & 0.063 & 0.114\end{array}\right]$.

The maximum eigenvalue of matrix $H_{1}$ is calculated to be 1.389, and the corresponding eigenvector $w_{1}=\left[\begin{array}{lll}0.9452 & 0.1563 & 0.2865\end{array}\right]$. Similarly, the corresponding eigenvectors of lane 2, lane 3 , and lane 4 are $w_{2}=\left[\begin{array}{lll}0.9106 & 0.0924 & 0.4028\end{array}\right], w_{3}=\left[\begin{array}{lll}0.8572 & 0.11590 .5018\end{array}\right]$, and $w_{4}=\left[\begin{array}{lll}0.9288 & 0.2028 & 0.3102\end{array}\right], \quad$ respectively. After normalizing each eigenvector and substituting it into equation (8), the traffic status comprehensive ranking of each lane as shown in Table 5 can be obtained.

Analyzing the development trend of the traffic operation status according to the rank of the last two periods, it can be seen that the traffic operation state of lane 1 and lane 4 is getting better, while lane 2 and lane 3 are showing a deteriorating trend. Combined with the traffic status classification of each lane, in this case, traffic congestion may occur in lane 2 and lane 3, especially lane 3. It is necessary to take precautions in advance and adopt certain traffic management and control measures for these two lanes to balance the traffic pressure of each lane, such as a driving ban for the truck, variable speed limits, temporary use of the emergency lane, or other measures for guiding vehicles to use the space resources of lane 1 and lane 4 rationally. 
Table 5: Traffic status comprehensive ranking of each lane.

\begin{tabular}{|c|c|c|c|c|c|c|c|c|}
\hline \multirow{2}{*}{ Time segment } & \multicolumn{2}{|l|}{ Lane 1} & \multicolumn{2}{|l|}{ Lane 2} & \multicolumn{2}{|l|}{ Lane 3} & \multicolumn{2}{|l|}{ Lane 4} \\
\hline & Evaluation value & Rank & Evaluation value & Rank & Evaluation value & Rank & Evaluation value & Rank \\
\hline $0 \sim 3 \mathrm{~min}$ & 0.313 & 1 & 0.379 & 2 & 0.596 & 3 & 0.382 & 2 \\
\hline $3 \sim 6 \mathrm{~min}$ & 0.327 & 2 & 0.367 & 1 & 0.441 & 1 & 0.410 & 3 \\
\hline $6 \sim 9 \min$ & 0.392 & 3 & 0.426 & 3 & 0.602 & 4 & 0.546 & 5 \\
\hline $9 \sim 12 \mathrm{~min}$ & 0.447 & 5 & 0.481 & 4 & 0.594 & 2 & 0.526 & 4 \\
\hline $12 \sim 15 \mathrm{~min}$ & 0.404 & 4 & 0.509 & 5 & 0.641 & 5 & 0.349 & 1 \\
\hline
\end{tabular}

\section{Conclusion}

The traffic characteristics of each lane of the eight-lane highway are analyzed in this paper, and a traffic status dynamic comprehensive evaluation method based on the lane level is proposed with the concept of "first classification then ranking." The state space division is used to classify the traffic operation status of each lane, and the principle of "difference-driven" is adopted to determine the rank of the traffic operation status and predict the development trend in real time. Compared with previous studies, the method proposed in this paper can better reflect the operating differences between lanes and detect congestion in advance, which provides the theoretical support for more refined management and control of eightlane highways.

However, there are several limitations in the present study. First, only traffic characteristics were considered when selecting evaluation indexes. Other factors such as driving behavior, traffic safety, and environmental pollution which will also make a complex impact on traffic status should be considered in the following studies. Second, the dynamic comprehensive evaluation method proposed in this paper applies to the basic sections of the multilane highway, but whether it is applicable to the diversion and merging areas needs further studies. In addition, the implementation conditions and effects of different lane management and control measures can be further studied according to the traffic operating differences between lanes so as to provide practical operation guidance for highway managers.

\section{Data Availability}

The traffic flow data used to support the findings of this study were supplied by Jiangsu Ninghu Highway Co., Ltd. Requests for access to these data should be made to Jiayan Shen (shenjiayanseu@163.com).

\section{Conflicts of Interest}

The authors declare that there are no conflicts of interest regarding the publication of this paper.

\section{Acknowledgments}

This research was supported by Major Science and Technology Special Project of the Transportation Department of Jiangsu Province, China (Z02). The authors are also grateful for the data support provided by Jiangsu Ninghu Highway Co., Ltd.

\section{References}

[1] Transportation Research Board, Highway Capacity Manual, Transportation Research Board of the National Academies (TRB), Washington, D.C., USA, 6th edition, 2016.

[2] N. Wu, "A new approach for modeling of fundamental diagrams," Transportation Research Part A: Policy and Practice, vol. 36, no. 10, pp. 867-884, 2002.

[3] B. S. Kerner, "Experimental features of self-organization in traffic flow," Physical Review Letters, vol. 81, no. 17, pp. 3797-3800, 1998.

[4] B. S. Kerner, "Experimental features of the emergence of moving jams in free traffic flow," Journal of Physics A: Mathematical and General, vol. 33, no. 26, pp. L221-L228, 2000.

[5] B. S. Kerner and S. L. Klenov, "Microscopic theory of spatialtemporal congested traffic patterns at highway bottlenecks," Physical Review E, vol. 68, no. 3, pp. 1-20, 2003.

[6] B. S. Kerner, "Three-phase traffic theory and highway capacity," Physica A: Statistical Mechanics and Its Applications, vol. 333, pp. 379-440, 2004.

[7] J. Xia and M. Chen, "Defining traffic flow phases using intelligent transportation systems-generated data," Journal of Intelligent Transportation Systems, vol. 11, no. 1, pp. 15-24, 2007.

[8] J. Xia and M. Chen, "A nested clustering technique for freeway operating condition classification," Computer-aided Civil and Infrastructure Engineering, vol. 22, no. 6, pp. 430437, 2007.

[9] M. Azimi and Y. Zhang, "Categorizing freeway flow conditions by using clustering methods," Transportation Research Record: Journal of the Transportation Research Board, vol. 2173, no. 1, pp. 105-114, 2010.

[10] C. Xu, P. Liu, W. Wang, and Z. Li, "Evaluation of the impacts of traffic states on crash risks on freeways," Accident Analysis and Prevention, vol. 47, no. 1, pp. 162-171, 2012.

[11] C. Xu, P. Liu, W. Wang, and Z. Li, "Identification of freeway crash-prone traffic conditions for traffic flow at different levels of service," Transportation Research Part A: Policy and Practice, vol. 69, pp. 58-70, 2014.

[12] E. R. Khansari, M. Tabibi, and F. Moghadas Nejad, "Lanebased car-following behaviour based on inductive loops," Proceedings of the Institution of Civil Engineers - Transport, vol. 170, no. 1, pp. 38-45, 2017.

[13] A. Duret, S. Ahn, and C. Buisson, "Lane flow distribution on a three-lane freeway: general features and the effects of traffic controls," Transportation Research Part C: Emerging Technologies, vol. 24, no. 5, pp. 157-167, 2012.

[14] C. L. Chu, N. Xie, X. Q. Chen et al., "Temporal-spatial analysis of traffic congestion based on modified CTM," Mathematical 
Problems in Engineering, vol. 2015, Article ID 136102, 11 pages, 2015.

[15] Q. F. Yang, R. R. Xing, L. L. Zheng et al., "Quantitative analysis of urban regional traffic status," Mathematical Problems in Engineering, vol. 2017, Article ID 2184397, 11 pages, 2017.

[16] Y. Q. Yang, S. M. Easa, Z. B. Lin et al., "Evaluating highway traffic safety: an integrated approach," Journal of Advanced Transportation, vol. 2018, Article ID 4598985, 11 pages, 2018.

[17] M. Hui, L. Bai, Y. B. Li et al., "Highway traffic flow nonlinear character analysis and prediction," Mathematical Problems in Engineering, vol. 2015, Article ID 902191, 7 pages, 2015.

[18] Y. Lv, Y. Duan, W. Kang et al., "Traffic flow prediction with big data: a deep learning approach," IEEE Transactions on Intelligent Transportation Systems, vol. 16, no. 2, pp. 865-873, 2015.

[19] N. G. Polson and V. O. Sokolov, "Deep learning for shortterm traffic flow prediction," Transportation Research Part C: Emerging Technologies, vol. 79, pp. 1-17, 2017.

[20] P. Cai, Y. Wang, G. Lu, P. Chen, C. Ding, and J. Sun, "A spatiotemporal correlative $\mathrm{k}$-nearest neighbor model for short-term traffic multistep forecasting," Transportation Research Part C: Emerging Technologies, vol. 62, pp. 21-34, 2016.

[21] B. Sun, W. Cheng, P. Goswami, and G. Bai, "Short-term traffic forecasting using self-adjusting k-nearest neighbours," IET Intelligent Transport Systems, vol. 12, no. 1, pp. 41-48, 2018.

[22] X. L. Luo, D. Y. Li, Y. Yang et al., "Spatiotemporal traffic flow prediction with KNN and LSTM," Journal of Advanced Transportation, vol. 2019, Article ID 4145353, 10 pages, 2019. 